\title{
Perspective
}

PERSPECTIVE Actualité en histoire de l'art

\section{2| 2019}

\section{Multiples}

\section{La carte postale, multiple documentaire du chef- d'œuvre}

The Postcard, a Documentary Multiple of the Masterpiece

Die Postkarte, ein dokumentarisches Multiple des Meisterwerks

La cartolina postale, multiplo documentario del capolavoro

La tarjeta postal, múltiple documental de la obra maestra

\section{Bertrand Tillier}

\section{(Q) OpenEdition}

\section{Journals}

Édition électronique

URL : http://journals.openedition.org/perspective/15875

DOI : $10.4000 /$ perspective. 15875

ISSN : 2269-7721

\section{Éditeur}

Institut national d'histoire de l'art

\section{Édition imprimée}

Date de publication : 30 décembre 2019

Pagination : 239-248

ISBN : 978-2-917902-50-9

ISSN : 1777-7852

\section{Référence électronique}

Bertrand Tillier, «La carte postale, multiple documentaire du chef-d'œuvre », Perspective [En ligne], 2 2019, mis en ligne le 30 juin 2020, consulté le 25 janvier 2021. URL : http://journals.openedition.org/ perspective/15875; DOI : https://doi.org/10.4000/perspective.15875 


\title{
La carte postale, multiple documentaire du chef-d'œuvre
}

\author{
Bertrand Tillier
}

À « l'ère de la reproductibilité technique » des œuvres d'art, il est une sorte d'image multiple dont Walter Benjamin, curieusement, ne souffle mot dans les versions successives de son essai, alors qu'il en fut lui-même l'expéditeur et le destinataire, ainsi que le montrent ses archives où elle figure ${ }^{1}$ - de la même manière qu'elle appartient aux planches du Bilderatlas Mnémosyne élaboré par Aby Warburg durant les années $1920^{2}$ (fig. 1) -, c'est la carte postale de reproduction des œuvres et objets d'art. Du début du XX $\mathrm{XX}^{\mathrm{e}}$ siècle jusqu'aux années 1970, principalement en Europe et aux États-Unis où étaient actifs les plus grands éditeurs, la carte postale photographique reproduisant des œuvres d'art - selon deux principales modalités, comme on le verra, soit dans un environnement comme les musées ou les Salons, soit en plan resserré - a constitué, en effet, une part non négligeable de cette imagerie de masse ayant succédé à l'imagerie populaire dominée par la gravure de reproduction qui avait contribué à préparer la société à son essor, comme l'ont avancé Claude Frère et Aline Ripert dans leur étude pionnière ${ }^{3}$. Significativement, Georges Goury déclarait dès 1901 :

\footnotetext{
La carte postale n'aura pas seulement été la renaissance de l'art de la petite estampe, elle aura un mérite de plus, celui d'avoir vulgarisé l'art. L'art, en effet, n'est plus le domaine de quelques privilégiés, qui, à coup de louis d'or, peuvent accumuler des chefs-d'œuvre ; par la carte postale, l'art est à tous ${ }^{4}$.
}

Cette production spécifique appartient donc pleinement à l'industrie culturelle majeure que constituèrent l'invention et l'acculturation de la carte postale illustrée dès la Belle Époque, et dont l'ambition était de documenter, d'inventorier et de représenter le réel, à travers des vues photographiques ${ }^{5}$. Mais, au début du $\mathrm{XX}^{\mathrm{e}}$ siècle, l'essor de la carte postale fut aussi une affaire économique, ainsi que l'a montré Marie-Ėve Bouillon dans sa thèse consacrée à l'éditeur Neurdein ${ }^{6}$ : en effet, les photographes professionnels y virent d'abord un moyen de contrebalancer leurs pertes dues à l'expansion de la photographie amateur, en créant de nouveaux débouchés corrélés au développement du tourisme. C'est dans ce contexte qu'Edmond Haraucourt, conservateur au Musée national du Moyen Âge, dans l'hôtel de Cluny, souhaitait que la carte postale, reproduite par procédé 


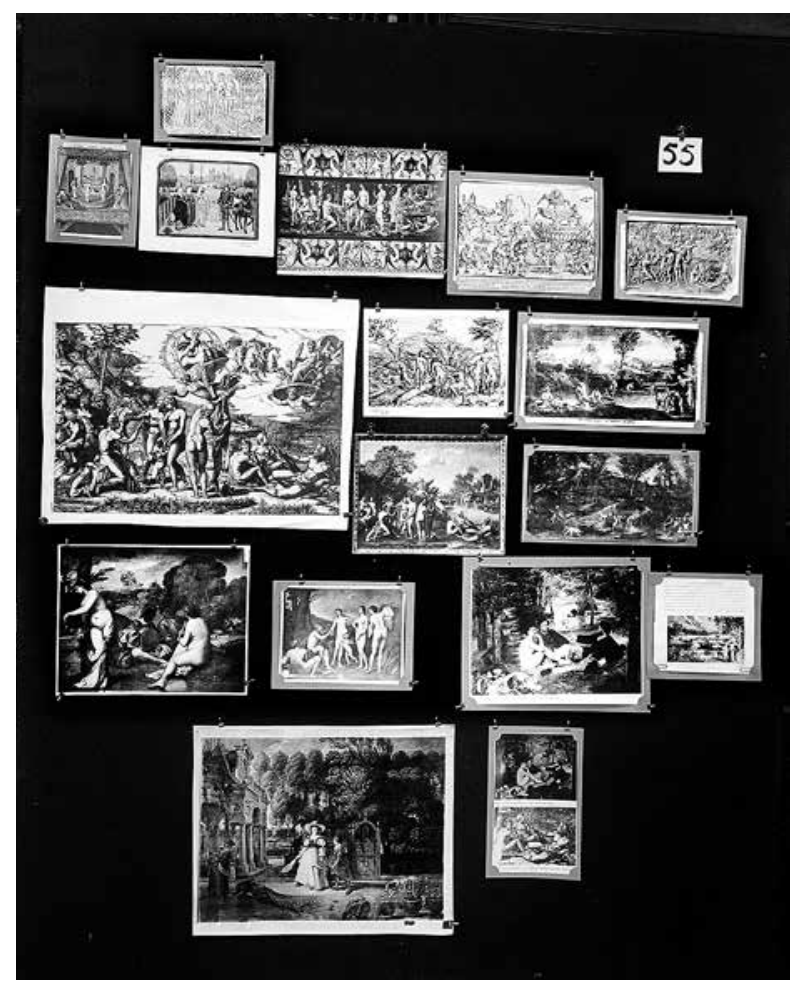

1. Aby Warburg, Bilderatlas Mnemosyne, planche 55, Londres, The Warburg Institute, University of London. phototypique, contribue à "vulgarise[r] les merveilles de la Nature et de l'Art ${ }^{7}$ ». De fait, dès la Belle Époque, on s'employa, selon un maillage spatial serré, à travers les cartes postales photographiques, à cataloguer le monde et à en diffuser des représentations, par le biais des vues de lieux (paysages et monuments), de figures et de mœurs pittoresques ou d'événements constitutifs de l'actualité politique et sociale. En regard de ces catégories génériques, et au-delà d'elles, il convient donc de se demander ce que sont les spécificités des cartes postales photographiques qui, durant une bonne part du $\mathrm{XX}^{\mathrm{e}}$ siècle, se sont attachées à reproduire des œuvres et objets d'art qu'elles ont démocratisés, tout en contribuant à les qualifier comme œuvres remarquables, voire comme chefs-d'œuvre. On s'interrogera aussi, entre production, circulation et réception, sur les usages éditoriaux et sociaux de ces multiples particuliers au sein d'une culture de masse.

En 1908, dans un article que publia une revue cartophile où il examinait « comment on collectionne les cartes postales ", Gaston Marcqfoy tentait d'opérer des classements dans la production pléthorique et hétéroclite de son temps, où les tirages pouvaient être

parfois vertigineux - de 5 à 10000 exemplaires d'une même carte chez les grands éditeurs comme Bergeret de Nancy qui, d'après ses propres dires, diffusait environ 50000 cartes par jour $^{8}$ - ou infinitésimaux mais incalculables. Il y définissait aussi cinq principales catégories fondées sur le succès des images les plus diffusées : les "cartes locales comprenant les vues, sites, monuments, types, mœurs, costumes, légendes [...], coutumes "; les " cartes fantaisie "; les " reproductions de tableaux et sculptures des musées et salons annuels "; les " actualités "; et les " cartes politiques" ". Sans savoir si son classement était fondé sur des chiffres décroissants de ventes, on remarque que Marcqfoy inscrit les cartes postales d'œuvres d'art en troisième position, entre les cartes dites de fantaisie, " représentant toutes sortes de sujets artistiques ${ }^{10}$ ", et celles relevant de l'actualité, avec lesquelles elles ne sauraient se confondre. En outre, que ces cartes jouissent d'une franche distinction catégorielle indique qu'elles satisfaisaient à un goût intrinsèque et à des ambitions propres, sanctionnés par des tirages significatifs et des usages spécifiques. En effet, plus explicitement que toutes les autres catégories, les cartes reproduisant des œuvres d'art correspondent à la vocation pédagogique qui fut celle des premiers grands éditeurs - Bergeret, Neurdein, Le Deley, Gilletta, Artaud, Combier, Yvon... - et des cartophiles originels. Dans un article de L'Humanité socialiste, où il tenait la rubrique d' " actualité artistique ", le critique et historien de l'art Léon Rosenthal ne manqua pas de souligner, en février 1914, combien " [p]ar la photographie, la carte postale illustrée, l'image dans un livre scolaire ou dans un album, le rayonnement de l'œuvre d'art s'accroît, l'action d'éducation nationale se décuple ${ }^{11}$ ». Il est significatif que 
le militant socialiste et défenseur de l'art social qu'était Rosenthal place la carte postale dans cet ensemble de matériels didactiques à destination de larges publics, et qu'il la convoque dans un article consacré à l'actualité que constituait alors le renouvellement, par le musée du Louvre, de la concession donnant depuis 1883, à la maison Braun, le droit de photographier les œuvres d'art de ses collections et d'en commercialiser les tirages $^{12}$. Depuis la fin du XIX ${ }^{\mathrm{e}}$ siècle, les musées nationaux - mais aussi les institutions plus modestes - avaient pris des dispositions, souvent contractuelles, dont la légitimité fut parfois contestée, pour que des opérateurs photographient leurs fonds dont les inventaires étaient, par ailleurs, conduits de plus en plus méthodiquement, et pour qu'ils en diffusent des reproductions en en gardant les bénéfices économiques, en contrepartie de leur investissement initial. C'est dans ce contexte que Braun obtint l'exclusivité des collections du Louvre contre la maison Goupil et contre les frères Neurdein qui lui intentèrent un procès ${ }^{13}$. Or les tensions s'aggravèrent encore entre les éditeurs de cartes postales qui, à la Belle Époque, tentèrent d'instaurer de véritables monopoles économiques à leur avantage, en signant des contrats d'édition et de vente avec les musées, comme l'ont montré Dominique Jarrassé et Emmanuelle Polack dans leur étude du corpus de 1600 cartes éditées par la maison Neurdein, entre 1904 et 1915, pour le Musée de sculpture comparée, en accord avec son conservateur, Camille Enlart, qui était lui-même un praticien de la photographie ${ }^{14}$.

Sur un double fond de démocratisation de l'art et de la culture et de massification des déplacements (mobilités économiques, conscription, congés, tourisme...), l'enjeu industriel est considérable, dès la Belle Époque et jusque dans la décennie 1970, car les divers usages des cartes postales assurent un important chiffre d'affaires aux éditeurs, tout en servant de formidable vecteur social de promotion aux institutions muséales et à leurs collections d'œuvres. Dès l'aube du XX⿳亠丷厂犬⿱ usages dans l'une de ses chroniques très ironiques du Mercure de France :

Il y a une chose qui m'amuse toujours en voyage, c'est le rôle de la carte postale illustrée. On dirait vraiment que la plupart des gens ne se déplacent que pour avoir le plaisir d'envoyer à leurs amis la photographie des sites ou des monuments rencontrés sur leur chemin. Je me souviendrai toujours de cette famille faisant irruption à la terrasse d'un hôtel d'où l'on avait sur la mer et les rochers une vue des plus pittoresques. Ils arrivèrent, jetèrent un coup d'œil au paysage, s'assirent résolument en lui tournant le dos et se mirent à signer et à timbrer à l'envers des cartes postales. Puis leur besogne maniaque achevée, ils disparurent par les rues de la petite ville. On s'est beaucoup moqué des Anglaises qui admirent les tableaux des musées dans les descriptions de leur Baedeker ou de leur Murray, on peut bien rire un peu des Françaises qui ne regardent les paysages que sur les cartes postales ${ }^{15}$.

À la suite de Gourmont, on pourrait s'amuser aujourd'hui des visiteurs de musées ou d'expositions temporaires passant plus de temps, dans la boutique, devant les présentoirs, à choisir des cartes postales d'œuvres que dans les salles, devant les œuvres elles-mêmes. On notera que ce pouvoir de diversion prêté à la carte postale d'œuvre, au détriment de son référent, avait été précédemment imputé au livret imprimé des collections accusé de monopoliser et détourner l'attention du visiteur en ouvrant une concurrence avec les artefacts qu'il était pourtant censé documenter ${ }^{16}$. Depuis l'orée du $\mathrm{XX}^{\mathrm{e}}$ siècle, que l'on désigne souvent comme "l'âge d'or de la carte postale ${ }^{17}$ ", la reproduction d'œuvres d'art par la carte postale est solidement établie, dont le succès culturel est dû à une imagerie standardisée, disponible en quantité et douée d'une fonction viatique où l'image a valeur de message - conformément à la théorie des médias de Marshall McLuhan, pour qui « le vrai message, c'est le médium lui-même, c'est-à-dire tout simplement les effets d'un médium sur l'individu ou la société18 ${ }^{18}-$, au point qu'une carte peut être 
envoyée par un épistolier à un correspondant sans autre rhétorique que quelques mots lapidaires ( "meilleur souvenir ", " bons baisers », etc.) relevant de la salutation ${ }^{19}$ ou d'autres formules d'une grande banalité (sur le lieu, la météo ou les activités), comme l'a rappelé Georges Perec dans un texte d'inspiration oulipienne ${ }^{20}$.

$\mathrm{Si}$, d'une part, grands et moins grands musées ont adopté la carte postale pour diffuser la reproduction de leurs chefs-d'œuvre ou de leurs collections plus modestes - avant 1914, dans l'entre-deux-guerres et durant les Trente Glorieuses, en noir et blanc, en sépia ou en couleurs -, et si, d'autre part, les grandes firmes et les marques locales, parisiennes ou provinciales, à diffusion régionale ou nationale voire internationale ${ }^{21}$, ont tôt et durablement consacré le musée et ses objets comme aussi incontournables que les édifices publics ou les sites naturels majeurs considérés comme remarquables qu'il faut donc avoir vus et que la carte-vue photographique contribue à donner à voir davantage dans un jeu de relations interpersonnelles -, cette imagerie industrielle n'est pas exclusivement destinée aux touristes ou aux voyageurs soucieux de faire partager leur expérience à des correspondants, ou d'emporter avec eux un souvenir de leurs visites. En effet, les cartes postales d'œuvres d'art intéressent, au-delà du grand public, toutes sortes d'autres acteurs sociaux qui en sont parfois de grands consommateurs : l'historien de l'art, l'archéologue et l'amateur d'art, dont les mobiles sont ceux de l'érudit ou du savant, désireux de se constituer une documentation visuelle et de capter un peu de l'aura perdue de l'œuvre d'art originale par le biais de sa reproduction mécanique conjuguée à sa " valeur d'exposition ${ }^{22}$ " démultipliée par la reproductibilité. C'est dans ces configurations que les cartes postales reproduisent des objets d'art et des chefs-d'œuvre uniques, dont elles font paradoxalement des multiples, en les éditorialisant d'une manière singulière, puisqu'il s'agit de reproductions à la fois réduites, hybrides et sérielles.

En effet, il convient de souligner, en premier lieu, combien la carte postale provoque une miniaturisation et une sorte de dématérialisation du référent qu'elle enchâsse dans sa matérialité propre et constante : un poids de 3 grammes et un format $14 \times 9 \mathrm{~cm}$. La réduction d'échelle s'accompagne d'une opération de condensation qui, dans le jeu du " régime scopique ${ }^{23}$ " et dans le champ des usages, introduit le mirage subreptice d'une maîtrise du réel que l'on peut embrasser d' " un seul coup d'œil ${ }^{24}$ " et tenir entre ses doigts - c'est-à-dire à portée de main -, que l'on est en mesure de soumettre à ses volontés d'élection et d'ambulation, et que l'on peut commenter ou s'approprier en y couchant ses propres mots et impressions. Il y a là une économie de la possession, dans tous les sens du terme, qui a sans nul doute pour vocation de rendre l'œuvre d'art préhensible et visible, par laquelle la carte postale procède du " modèle réduit " tel que Claude LéviStrauss l'a étudié, à la fois " chef-d'œuvre » et " type même de l'œuvre d'art ${ }^{25}$ ". Ces qualités sont liées au statut hybride de la carte postale, en tant qu'objet icono-textuel ${ }^{26}$. Dispositif visuel, la carte postale est la reproduction photographique d'une qualité et d'une précision variables, qui, dès lors qu'elle s'empare de l'œuvre d'art, la reproduit tantôt en cadrage serré et en gros plan - à fond perdu ou sur sa cimaise partiellement visible -, tantôt in situ, c'est-à-dire dans son environnement spatial direct, au sein de l'institution qui la conserve : la salle du musée ou le pan de mur où elle s'intègre à un dispositif d'accrochage ou d'installation combinant d'autres objets. Selon l'une ou l'autre de ces configurations, l'œuvre d'art reproduite par la carte postale est acquise et lue soit comme un objet autonome, soit comme un élément métonymique de la collection muséale, sans qu'on parvienne alors à clarifier si elle cristallise intrinsèquement l'attention ou si elle joue le rôle d'intermédiaire dans une approche élargie. Mais la carte postale est aussi un objet textuel, par les légendes qui sont adjointes à la reproduction photographique - au recto sur le fond même de l'image, dans ses marges (inférieures, supérieures ou latérales) 
ou dans une sorte de cartouche ; puis au verso, à partir des années 1920. Quels que soient les cas de figure et les combinaisons, l'appareil textuel de la carte postale explicitant l'œuvre d'art reproduite s'apparente ici à la forme du $\operatorname{cartel}^{27}$, tel que les musées ont commencé à l'adopter à la flexion des décennies $1830-1840^{28}$, pour rendre accessibles les principaux éléments d'identification des œuvres aux visiteurs des musées, tout en les dispensant d'acquérir les livrets et les catalogues des collections. Déplaçable, ce cartel de l'œuvre qui ne dit pas son nom dans la carte postale, puisqu'il sert de légende à l'image, participe d'un mouvement plus général et croissant de classement, d'identification et de valorisation scientifique des collections des musées soucieux d'éduquer les visiteurs, qu'amplifient les éditeurs de cartes postales photographiques attentifs à renseigner leurs clients pour leur édification propre ou celle de leurs destinataires. En indiquant, en regard de la photographie d'un tableau ou d'une sculpture, et même pour les chefs-d'œuvre qui en auraient moins besoin, son auteur, son titre, sa date, sa technique et sa localisation - parfois augmentés d'un commentaire contextuel, d'une anecdote ou d'une ekphrasis -, les cartes postales reproduisant des œuvres d'art fonctionnent comme autant de seuils, aux sens que Gérard Genette a donnés à ce terme dans son essai éponyme ${ }^{29}$ : des lisières ou des vestibules de l'image, qui l'entourent, la présentent et en conditionnent la réception et la consommation, par des effets de transition et de transmission, et par des dynamiques de transaction entre l'éditeur et le spectateur-lecteur. Les cartes postales rendent cette prouesse possible, que peu d'autres types de reproductions mécaniques parviennent à accomplir : il s'agit d'un support autonome et bon marché où sont associés et rendus indissociables l'œuvre d'art et son cartel, là où le livre, le guide ou le périodique ne parviennent pas toujours à concilier ces exigences avec autant d'efficacité. Enfin, la carte postale s'inscrit dans un dispositif sériel qui ne doit pas être mésestimé. Dotée d'une racine de titre thématique - "Collections du Musée du Louvre ", "Chefs-d'œuvre des musées de Berlin », " Sculptures du Moyen Âge ", "L'histoire de Paris au musée Carnavalet ", " La peinture dans les musées de province "... - et flanquée d'un numéro la situant dans la série à laquelle elle appartient, chaque carte postale constitue une livraison d'un plus vaste ensemble feuilletonnant qui n'interdit toutefois pas qu'on l'en distraie pour la consommer à l'unité. Toutefois, une carte postale produite au sein d'une série demeure susceptible d'être ressaisie, du point de vue de sa réception, selon une dynamique déterminant sa signification par une "poétique de la sérialité " propre aux logiques de consommation de la culture populaire et médiatique moderne, telles que Matthieu Letourneux les a interrogées ${ }^{30}$. Dans le même ordre d'idée, la carte postale reproduisant les œuvres d'art peut aussi appartenir à des carnets dédiés à une localité, un musée, un thème, une période historique ou un artiste, documentés à la manière d'un reportage visuel, d'où l'on peut détacher - suivant les pointillés - chaque vue photographique pour l'isoler et l'expédier, mais que l'on acquiert aussi tels de petits albums, presque des livres ${ }^{31}$, et que l'on peut conserver dans leur intégrité, comme l'y invitent les éclatantes couvertures de ces recueils de cartes, avec leurs recherches de composition graphique, de typographie, de gaufrage et d'impression polychrome, d'une exécution souvent très soignée.

$\mathrm{Au}$ fond, dans cette configuration de productions et d'usages, la carte postale d'œuvre d'art est toujours un multiple documentaire qui participe de la promotion des chefs-d'œuvre établis - c'est encore davantage le cas pour celles du musée de Sculpture comparée qui valorise des reproductions de copies de chefs-d'œuvre ${ }^{32}-$ ou qui contribue à en fabriquer d'autres, plus ou moins connus, en attirant l'attention du spectateur sur des œuvres choisies - et en plaçant en situation l'objet reproduit et représenté (c'est-à-dire rendu présent et d'une présence réitérée), dans un régime du pittoresque permanent qui est celui de la carte postale, invitant son destinataire à la découverte et au voyage, par la délégation de l'objet viatique. 


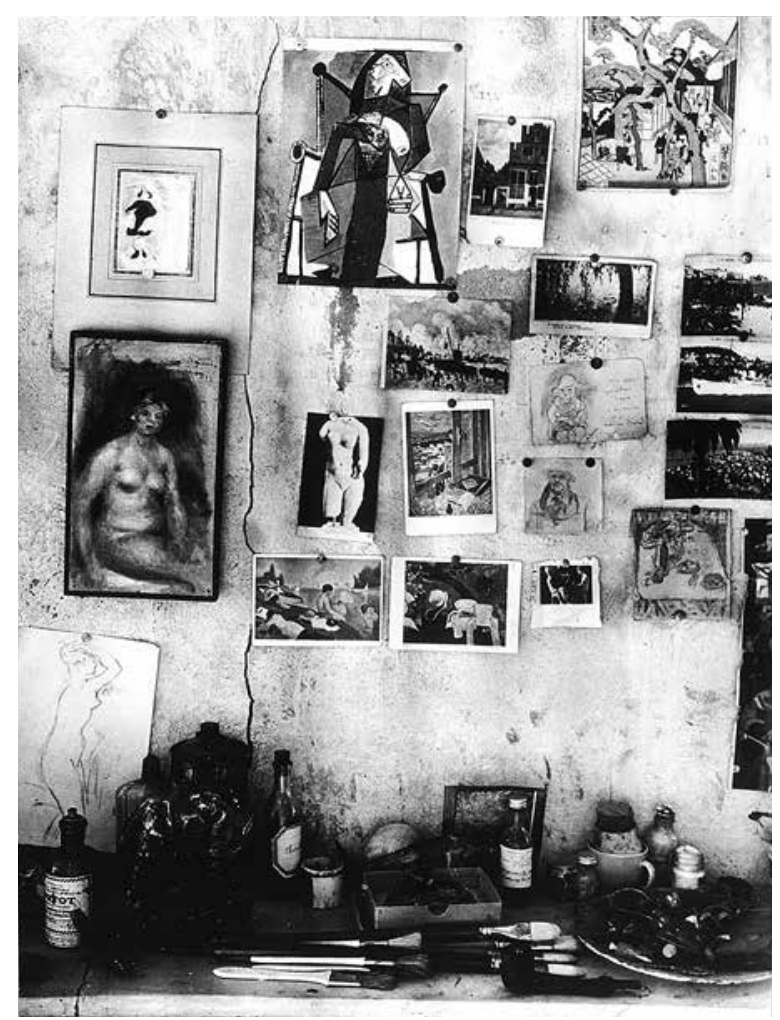

2. Brassaï (Halasz Gyula), Mur chez Bonnard en 1946, photographie en collection particulière.
Si la carte postale est un objet dont la vocation initiale est celle $d^{\prime}$ « un imprimé sur un support semi-rigide destiné à un usage postal, pour une correspondance à découvert ", selon la définition canonique d'Albert Thinot ${ }^{33}$, ses usages sociaux sont multiples qui outrepassent la stricte sphère épistolaire et enserrent les reproductions d'œuvres d'art dans un vaste champ d'intentions et de pratiques. Choisie avec attention et intention, acquise pour être expédiée à un correspondant que l'on veut dépayser, surprendre ou éveiller, la carte postale est souvent conservée ; elle peut même être acquise en vue de sa conservation. Elle est alors " dépouillée de son actualité, mais encore intelligible ${ }^{34} "$, pour entrer dans ce que Marie-Thérèse Duflos-Priot a appelé son " deuxième âge ${ }^{35}$ " - « l'âge de l'album ou du trésor personnel ${ }^{36} "$. Dans l'un des premiers ouvrages consacrés à l'histoire de la carte postale, Georges Guyonnet notait en 1947 : « De 1900 à 1914, il n'y eut guère de foyer en France qui ne possédât son album où s'alignaient soigneusement les cartes reçues aux jours fastes de la famille ${ }^{37}$ ". Sans doute des cartes postales de chefs-d'œuvre ont-elles été conservées dans ce contexte, parmi les paysages spectaculaires et les monuments emblématiques. Mais celles-ci, une fois leur "message dévitalisés ${ }^{38}$ ", sont surtout devenues des objets de collectionneurs qui les expertisent, les cotent, les revendent et les rachètent parfois vierges de toute correspondance postale -, pour les intégrer dans de nouveaux ensembles, définis selon des critères et des mobiles très différents, en les ordonnant dans des boîtes ou des albums thématiques dédiés. Les cartes sont ainsi rebattues, selon des centres d'intérêt, des modalités de lecture et de regard introduisant des contraintes qui conditionnent la démarche des cartophiles dont l'apparition est contemporaine de l'avènement de leur objet ${ }^{39}$. On collectionne ainsi les cartes postales reproduisant les œuvres du Salon de la Société des Artistes français ou de la Société nationale des Beaux-Arts, celles du Musée national du Moyen Âge ou du musée Carnavalet. Mais on peut aussi choisir de collectionner les cartes de peintures d'histoire ou de paysage, de natures mortes ou de portraits, de vases grecs ou de sculptures antiques et, par un filtre supplémentaire, ne s'intéresser qu'à celles produites par un éditeur - Bergeret, Neurdein ou Combier, par exemple. Il y a là une forme d'appropriation qui a pour dessein de constituer une nouvelle collection - une collection puisée, à la façon d'un " univers de consolation ", selon le concept théorisé par Claude Poliak ${ }^{40}$, dans la (ou les) collection(s) des institutions dont le patrimoine se soustrait fondamentalement à toute possibilité de possession individuelle -, qui n'est plus celle des musées à laquelle appartiennent les œuvres d'art reproduites et qui n'est plus tout à fait non plus celle des séries de l'éditeur.

Dans ces opérations appropriatives où la carte postale est un substitut éditorial de

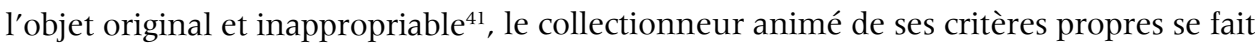


conservateur d'un musée de papier, à sa main, sa mesure et son goût. Il en résulte des choix et des absences - par éviction ou par contrainte -, des lieux et des dispositifs (l'album, le classeur, la boîte, le tiroir...), des effets de montage et de narration par juxtaposition - comme dans la peinture de Robert Delaunay, dont Pascal Rousseau a exploré la complexité ${ }^{42}-$, sur le principe des panneaux préparatoires à l'Atlas Mnémosyne de Warburg ou du mur où on les punaise, à la manière de Bonnard dans son atelier photographié par Brassaï en 1946 (fig. 2). Par ces usages appropriatifs, dont les motivations peuvent être diverses, la carte postale de chef-d'œuvre devient ainsi l'objet de "musées imaginaires ", dont le collectionneur est le commandeur et où il construit son récit de l'histoire de l'art, en montant les images en regard les unes des autres. La collection de reproductions, "musée sans murs ", selon l'expression de Georges Didi-Huberman ${ }^{43}$, devient ainsi un espace de lecture, de dialogue et de confrontation. Les usages personnels et professionnels de la carte postale d'œuvre d'art par Le Corbusier sont éloquents à cet égard, comme l'indiquent les archives du peintre-architecte ${ }^{44}$. Le Corbusier posséda plus de 2000 cartes postales qui lui furent « un instrument de dialogue interne, [...] une relation intime et vitale avec le monde qui l'entoure ${ }^{45}$ ", dont Luis Burriel Bielza a montré les différents mécanismes d'appropriation documentaire (fig. 3). En effet, l'artiste explora dans les cartes qu'il conserva - qu'il les ait reçues ou acquises les ressources iconographiques et formelles questionnées et intégrées dans son œuvre propre, par des annotations manuscrites ou graphiques, des duplications et des citations qui cherchent à ausculter « un phénomène de concordance [...] - véritable manifestation d'acoustique plastique ", conforme à sa théorie de l' "Espace indicible ${ }^{46}$ ".

Le chef-d'œuvre rendu reproductible par la carte postale radicalise, en somme, le rapport d'André Malraux à la photographie, dont les ressources sont explorées dans les éditions successives du Musée Imaginaire (1947, 1951, 1965) fondé, comme on le sait, sur une opposition avec l'incomplétude définitive des " vrais musées " constitués de " la réunion de tant de chefs-d'œuvre, d'où tant de chefs-d'œuvre sont absents ${ }^{48}$ " et que

3. "Potsdam. Sans souci. Neues Palais » [recto] et texte et dessin de la coupole par Le Corbusier [verso], carte postale de la collection de l'architecte, Paris, Fondation Le Corbusier.
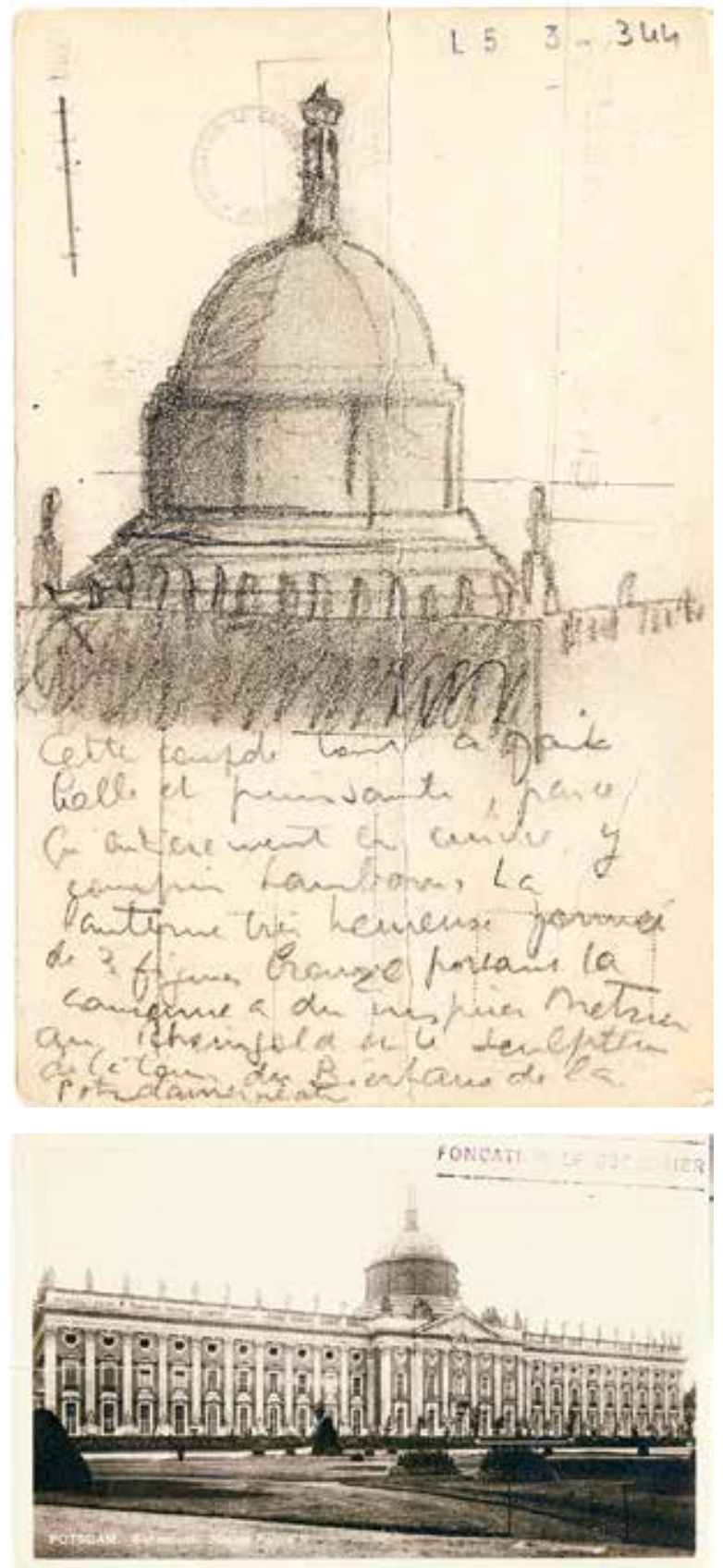
complète opportunément le voyage, sans jamais pouvoir en combler les lacunes. En écho à Malraux, pour qui « le Musée Imaginaire de chacun $^{49}$ " repose sur le pouvoir de révélation et d'expressivité de la photographie, édifiant une histoire de l'art conçue comme " l'histoire de ce qui est photographiable ${ }^{50}$ ", la reproductibilité et la sérialité de la carte postale placent les collections d'images sous le signe d'une gouverne et d'un goût individuels, qui créent des rapprochements - non seulement entre les ouvres reproduites, mais aussi entre celles-ci et les spectateurs de leurs reproductions, pour former de nouveaux "systèmes des objets " jadis théorisés par Jean Baudrillard ${ }^{51}$ - et des nouveaux lieux physiques ou mentaux, documentaires et intimes. Fabriquées en série, diffusées à grande échelle et consommées avec avidité, les cartes postales photographiques d'œuvres d'art font masse. Pourtant, par leur élection, elles s'inscrivent dans l'existence de leur propriétaire qui les extirpe de leur état de marchandise, en les singularisant par leur personnalisation. La présence auratique des œuvres et la connaissance de ces dernières y forment à la fois un " montage propédeutique ", des " albums de la famille de l'art universel " et des " trophées personnels d'un grand voyageur dans l'univers des formes ${ }^{52}$ ". De la sorte, par leurs modes de diffusion, de dissémination et de consommation, les cartes postales d'œuvres d'art - objets codifiés jusqu'à la standardisation, représentations multiples et sérielles - ne cessent de s'actualiser dans le regard de tout individu s'en emparant ${ }^{53}$, selon le syndrome de la relique ou du fétiche, dont les usages sont porteurs d'interprétations subjectives et divergentes, parfois en contradiction flagrante avec leurs conventions de production, les menant à la lisière d'une redéfinition. C'est ainsi que les cartes postales reproduisant des œuvres et des objets d'art finissent par constituer une archive de leur référent original - une archive, au sens où Michel Foucault l'entendait ${ }^{54}-$, constituée des strates successives dans l'accumulation desquelles se détermine, selon des cadres culturels, sociaux ou économiques toujours changeants, la signification réactualisée de l'œuvre. Les recouvrements plus ou moins opaques et les effacements partiels, auxquels Antonio Saura se livre, à l'encre noire, sur des cartes postales de chefs-d'œuvre de Goya ou de Picasso ${ }^{55}$, montrent à quel point cette catégorie d'objets reproductibles et multiples qui appartient à la culture de masse et au champ des ephemera ${ }^{56}$ résiste à sa disparition en n'échappant jamais aux modalités d'appropriation qui les singularisent. En effet, en dépit de leur stabilité matérielle, ces images multiples d'œuvres singulières que sont les cartes postales de chefs-d'œuvre accèdent au statut $\mathrm{d}^{\prime}$ " objets en devenir ${ }^{57}$ " , dont les usages et les lectures construisent, par enchevêtrements infinis ${ }^{58}$, des formes d'attachement et de médiation les investissant d'une fonction de témoignage et d'une valeur testimoniale. 


\section{Bertrand Tillier}

Historien de l'art, Bertrand Tillier est professeur $\mathrm{d}^{\prime}$ 'histoire contemporaine à l'université Paris 1 - Panthéon-Sorbonne, où il enseigne la culture visuelle et I'histoire des médias. Ses travaux portent, pour partie, sur l'image imprimée et ses usages sociaux. Il dirige les Éditions de la Sorbonne.

\section{NOTES}

1. Ursula Marx, Florent Perrier, Michael Schwartz, Gudrun Schwarz, Erdmut Wizisla (dir.), Walter Benjamins Archive. Bilder, Texte und Zeichen, cat. exp. (Berlin, Akademie der Künste, 2006), Berlin, Suhrkamp Verlag, 2006.

2. Aby Warburg, L'Atlas Mnémosyne, avec un essai de Roland Recht, Paris, L'écarquillé / INHA, 2012.

3. Claude Frère et Aline Ripert, La carte postale, son histoire, sa fonction sociale, Paris, Éditions du CNRS, (1983) 2001, p. 174

4. Georges Goury, "Allocution du président de l'APN", dans Revue illustrée de la carte postale, ${ }^{\circ} 24$, décembre 1901, p. 248.

5. Sur l'histoire de la carte postale, la bibliographie est pléthorique. On mentionnera Frank Staff, The Picture Postcard \& its Origins, Londres, Lutterworth Press, 1966 ; Frère et Ripert, (1983) 2001, cité n. 3 ; Daniel Bénard et Bruno Guignard, La carte postale des origines aux années 1920, Saint-Cyr-sur-Loire, Éditions Alan Sutton, 2010 ; Lynda Klich et Benjamin Weiss (dir.), The Postcard Age: Selections from the Leonard A. Lauder Collection, Boston, Museum of fine arts, 2012.

6. Marie-Ève Bouillon, Naissance de l'industrie photographique, Les Neurdein, éditeurs d'imaginaires, 1863-1918, thèse de doctorat sous la direction d'André Gunthert et Christophe Prochasson, Paris, EHESS, 2017.

7. Cité dans Frère et Ripert, (1983) 2001, cité n. 3, p. 28.

8. Dans Le Figaro illustré, octobre 1904. Les tirages sont difficiles à établir avec précision, en l'absence d'archives commerciales.

9. Gaston Marcqfoy, «Comment on collectionne les cartes postales ", dans La Diane, Revue artistique de la carte postale, $n^{\circ} 2,15$ octobre 1908, p. 3.

10. Ibidem.

11. Léon Rosenthal, « La photographie et le musée du Louvre ", dans L'Humanité, "L'Actualité artistique", 17 février 1914, p. 4 (repris dans Léon Rosenthal, Chroniques d'art de l'Humanité, 1909-1917, édition établie par Vincent Chambarlhac, Thierry Hohl et Bertrand Tillier, Dijon, Éditions universitaires de Dijon [coll. "Sources »], 2012, p. 128).

12. Voir le catalogue de l'exposition: Ulrich Pohlmann et Paul Mellenthin (dir.), Adolphe Braun, Une entreprise photographique européenne au 19e siècle, Jean-Léon Muller, Chantal Philippe (trad. fra.), cat. exp. (Colmar, musée Unterlinden, 2018), Munich, Shirmer / Mosel Verlag, 2018, p. 219-232.
13. Voir Pierre-Lin Renié, "Braun versus Goupil et quelques autres histoires, la photographie au musée du Louvre au XIX siècle ", dans État des lieux 2, Bordeaux, musée Goupil, 1999, p. 97-151; et sur le différend Neurdein-Braun : Bouillon, 2017, cité n. 6.

14. Dominique Jarrassé et Emmanuelle Polack, «Le musée de Sculpture comparée au prisme de la collection de cartes postales éditées par les frères Neurdein (1904-1915) ", dans Les Cahiers de l'École du Louvre, $\mathrm{n}^{\circ} 4,2014$, p. 2-20 [en ligne, URL : https://journals. openedition.org/cel/476 (consulté le 28 mars 2019)].

15. Remy de Gourmont, Je sors d'un bal paré, Paris, E. Champion (coll. "Les Amis d'Édouard ", 6), 1912 (repris dans Remy de Gourmont, Les arts et les ymages, Bertrand Tillier [éd.], Saint-Sébastien-sur-Loire, Séquences, 2006, p. 172-173).

16. Chantal Georgel, «Petite histoire des livrets de musée ", dans Eadem (dir.) La jeunesse des musées, Les musées en France au XIX siècle, cat. exp. (Paris, musée d'Orsay, 1994), Paris, Réunion des musées nationaux, 1994, p. 207-214.

17. Ado Kyrou, Lâge d'or de la carte postale, Paris, Balland, 1966. Cette expression - problématique, puisqu'elle cantonne l'expansion de la carte postale à la Belle Époque, en occultant la vitalité de cette production jusque dans les années 1970 - court à haute fréquence dans la bibliographie sur la carte postale, où les travaux émanent souvent de collectionneurs dont les propos ne sont, par ailleurs, pas dénués de nostalgie et s'emploient à mythifier un objet dit " des origines ».

18. Marshall McLuhan, Pour comprendre les médias, Les prolongements technologiques de l'homme (1964), Jean Paré (trad. fra.), Paris, Éditions du Seuil (coll. « Points Essais »), 2005, p. 25.

19. Marie-Thérèse Duflos-Priot, «Au mur d'un bistrot : analyse d'un corpus de cartes postales ", dans Ethnologie française, t. 8, n 1, 1978, p. 71-82 [en ligne, URL : https: //www.jstor.org/stable/40988474 (consulté le 2 avril 2019)].

20. Georges Perec, « 243 cartes postales en couleur véritables» (1978), repris dans Georges Perec, L'infraordinaire, Paris, Éditions du Seuil (coll. "La Librairie du XXe siècle »), 1989, p. 9-13.

21. Bergeret, Humblot et Simon ou Royer à Nancy, Solyphot à Lyon, Braun ou Neurdein à Paris, Jack à Louannec, Le Trèfle à Cognac, Combier à Mâcon...

22. Walter Benjamin, "L'œuvre d'art à l'époque de sa reproductibilité technique » (1939), dans CEuvres, III, Maurice de Gandillac, Rainer Rochlitz (trad. fra.), Paris, Gallimard, 2000, p. 282

23. Jonathan Crary, L'art de l'observateur, Vision et modernité au XIXe siècle, Nîmes, Jacqueline Chambon (coll. «Rayon Photo »), 1994 [éd. orig. Techniques of the Observer : on Vision and Modernity in the Nineteenth Century, Cambridge, Mass., The MIT Press, 1990].

24. Claude Lévi-Strauss, La Pensée sauvage (1962), Paris, Presses Pocket (coll. « Agora »), 1990, p. 37-38.

25. Ibidem, p. 37. Pour Lévi-Strauss, il s'agit du « chefd'œuvre du compagnon »; on élargit donc ici la notion.

26. Selon le concept forgé par Michael Nerlich, voir 
"Qu'est-ce qu'un iconotexte? », dans Alain Montandon (dir.), Iconotextes, Paris, Ophrys, 1990 p. 255-302.

27. Isabelle Cahn, "Cadres et cartels ", dans Georgel, 1994, cité n. 16, p. 225-229.

28. Cahn, 1994, cité n. 27, p. 221-229.

29. Gérard Genette, Seuils (1987), Paris, Éditions du Seuil (coll. « Essais »), 2002.

30. Matthieu Letourneux, Fictions à la chaîne, Littératures sérielles et culture médiatique, Paris, Éditions du Seuil (coll. «Poétique »), 2017.

31. Nicolas Petit, L'Éphémère, l'occasionnel et le nonlivre à la bibliothèque Sainte-Geneviève (XV-XVIII siècles), Paris, Klincksieck, 1997.

32. Jarrassé et Polack, 2014, cité n. 12.

33. Cité par Sébastien Lapaque, Théorie de la carte postale, Arles, Actes Sud, 2014, p. 28.

34. Duflos-Priot, 1978, cité n. 19, p. 78.

35. Ibidem.

36. Ibidem

37. Georges Guyonnet, La Carte postale illustrée, son histoire, sa valeur documentaire, Paris, Chambre syndicale de la carte postale illustrée, sd [1947], p. 19

38. Duflos-Priot, 1978, cité n. 19, p. 78

39. En effet, dès le début du XX⿳亠丷厂 siècle, et à l'échelle internationale, naissent les cercles d'amateurs et leurs revues et bulletins spécialisés : le Cartophile Club en France, The Picture postcard en Angleterre, La Cartolina postale illustrata en Italie, La Ispaña cartofila en Espagne... Voir Frère et Ripert, (1983) 2001, cité n. 3, p. 28.

40. Claude Poliak, Aux frontières du champ littéraire, Sociologie des écrivains amateurs, Paris, Economica, 2006.

41. Selon Bataille : "Les biens de la culture ne peuvent en réalité être l'objet d'aucune appropriation particulière » (Georges Bataille, "L'équivoque de la culture » (1956), dans OEuvres complètes, Paris, Gallimard, 1988, t. XII, p. 442).

42. Pascal Rousseau, Robert Delaunay, L'invention $d u$ pop, Paris, Hazan, 2019.

43. Georges Didi-Huberman, L'Album de l'art à l'époque du "Musée imaginaire », Paris, Hazan / musée du Louvre éditions (coll. "La chaire du Louvre »), 2013, p. 20.

44. Luis Burriel Bielza (dir.), Le Corbusier, La passion des cartes, cat. exp. (Bruxelles, CIVA, 2013), Bruxelles, CIVA / Éditions Mardaga, 2013.

45. Ibidem, p. 21.

46. Cité par Burriel Bielza, 2013, cité n. 44, p. 31.

47. André Malraux, Le Musée Imaginaire (1965), Paris, Gallimard (coll. "Folio essais »), 1996.

48. Ibidem, respectivement p. 16 et 13 .

49. Ibidem, p. 252.

50. Ibidem, p. 123

51. Jean Baudrillard, Le système des objets (1968), Paris, Gallimard (coll. « Tel »), 1990.

52. Didi-Huberman, 2013, cité n. 43, respectivement p. 68 et 82 .
53. Letourneux, 2017, cité n. 30, p. 60

54. Michel Foucault, L'Archéologie du savoir, Paris, Gallimard (coll. « Bibliothèque des Sciences humaines »), 1969.

55. Didier Semin, Antonio Saura, Montages, 1956-1996, Paris, Galerie Lelong, 2012. Voir le colloque organisé en 2013 sous la direction de Françoise Levaillant, "Carte postale et création, Usages, fonctions et enjeux de la carte postale dans le champ artistique (XIXe-XXI siècles)", Besançon, musée du Temps / Paris, INHA, 2013 (actes non parus), voir, en ligne, URL : http:// www.centrechastel.paris-sorbonne.fr/actualites/cartepostale-et-creation.

56. Ruth E. Iskin, Britany Slasbury (dir.), Collecting Prints, Posters and Ephemera, Perspectives in a Global World Londres / Oxford / New York, Bloomsbury (coll. « Visual Arts »), 2019.

57. Thierry Bonnot, L'Attachement aux choses, Paris, CNRS éditions, 2014.

58. Ian Hodder, Entangled. An Archeology of the Relationships between Humans and Things, Malden Mass., Wiley Blackwell, 2012. 